\title{
Clinical manifestations associated with novel mutations in the coiled-coil domain of STAT1
}

\author{
Amit Nahum ${ }^{\mathrm{a} *}$ and Ilan Dalal ${ }^{\mathrm{b}}$
}

\begin{abstract}
Monoallelic mutations in STAT1 are associated with a variety of clinical patterns. We studied patients with novel mutations in the coiled-coil domain of STAT1. We found that clinical manifestations can vary from mild Candida infections limited to the oropharyx to serious serial strokes and skin cancer. Autoimmune manifestations were found to be rare and limited to hypothyroidism. Immune evaluations were normal or near normal in all patients with the exception of anergy to Candida. Mutation in the coiled-coil domain results in susceptibility to mucus membrane candidiasis as well as brain vascular anomalies.
\end{abstract}

Statement of novelty: We describe novel mutations in the coiled-coil domain of STAT1.

\section{Introduction}

Signal transducer and activator of transcription 1 (STAT1) plays a central role in signaling responses to a wide variety of cytokines including interferon (IFN)$\alpha,-\beta$, and $-\gamma$. Transcription 1 (STAT1) plays a central role in signaling response. Cytoplasmic STAT1 is activated by tyrosine phosphorylation, mostly by the Janus family of kinases (JAK), resulting in translocation to the nucleus and binding to specific DNA sequences (O'Shea et al. 2013).

Mutations in STAT1 have been reported in several phenotypically different immunodeficiencies such as the Mendelian susceptibility to mycobacterial diseases with susceptibility to mycobacteria as well as viral and fungal diseases (Boisson-Dupuis et al. 2012). The complete form of STAT1 deficiency is fatal in early life (Dupuis et al. 2003). Autosomal dominant gain of function mutations in the coiled-coil domain of STAT1 were recently reported, and they were associated with the clinical phenotype of chronic mucocutaneous candidiasis
(CMCC), autoimmune phenomena, and central nervous system vascular events (Liu et al. 2011; van de Veerdonk et al. 2011). We recently reported an association between heterozygous STAT1 mutations, which are caused by STAT1 loss of function defects, with a phenotype of progressive severe combined immunodeficiency manifested by a decline in $\mathrm{T}$ and $\mathrm{B}$ lymphocyte numbers and function (Sharfe et al. 2014). These mutations are found in the DNA binding domain and in close proximity to that area.

We present a group of patients with novel mutations in the coiled-coil domain. These patients show severe central nervous system vascular morbidity, autoimmune manifestations, and recurrent sinopulmonary infections and autoimmunity in some patients.

\section{Methods}

\section{Flow cytometry}

Peripheral blood mononuclear cells were obtained by Ficoll-Hypaque density gradient centrifugation, and

\footnotetext{
a Department of Pediatrics A Soroka, University Medical Center, Ben Gurion University of The Negev, Beer Sheva, Israel; ${ }^{b}$ Pediatric Allergy and Immunology Unit, E. Wolfson Medical Center, Holon and Tel-Aviv University, Tel-Aviv, Israel.

${ }^{*}$ Corresponding author: Amit Nahum/AmitNa@clalit.org.il
}

Submitted 2 October 2014

Accepted 7 October 2014

Available online 9 October 2014

LymphoSign Journal 1:97-103 (2014)

dx.doi.org/10.14785/lpsn-2014-0021 
surface phenotypes were determined by flow cytometry on a Coulter EPICS V flow cytometer (Coulter). All fluorescent labelled antibodies were purchased from eBiosciences (San Diego, Calif., USA).

\section{Serum concentration of immunoglobulin and specific antibodies}

Serum concentrations of immunoglobulins were measured by nephelometry. Serum IgE concentration was measured using radioimmunoassay with the IgE PRIST kit (Pharmacia Diagnostics, Dorval, Que., Canada). Levels of serum antibodies to tetanus were measured using ELISA, and polio antibody titres were determined with complement fixation.

\section{T- and B-cell proliferative response}

Lymphocyte proliferative responses to mitogens (including phytohemagglutinin and anti-CD3 antibodies) and to a panel of recall antigens (including Candida, tetanus, herpes zoster, and cytomegalovirus) were determined by thymidine incorporation. All assays were performed in triplicate and were compared with simultaneously stimulated normal controls.

\section{Sequencing analysis}

Patients' genomic DNA was amplified with polymerase chain reaction (PCR) and sequenced for the STAT1 gene. Primers were designed to amplify fragments up to $60 \mathrm{bp}$ upstream and downstream of each transcript exon of the STAT1 gene. PCR conditions consisted of $94{ }^{\circ} \mathrm{C}$ for $30 \mathrm{~s}, 55^{\circ} \mathrm{C}$ for $30 \mathrm{~s}$, and $68{ }^{\circ} \mathrm{C}$ for $2 \mathrm{~min}$, for a total of 35 cycles using Elongase (Invitrogen) as previously described (Sharfe et al. 2014). PCR products were electrophoresed on $0.8 \%$ agarose gels. Each PCR fragment was subsequently cut, and purified fragments were sequenced using a DTCS Quick Kit on an automated sequencer (BeckmanCoulter CEQ 8000).

\section{Results}

\section{Patients}

\section{Case 1, Family 1}

A 43-year-old man was followed from early childhood because of persistent oral thrush, extensive skin lesions, and distinguishing nail dystrophy. Partial improvement was obtained with continuous ketoconzale. At the age of 15 years, he had a sudden onset of slurred speech, facial paresis, as well as left hemiplegia. Angiography showed multiple dilatations of major arteries (Figure 1). There was also diminished foiling of some branches of the middle anterior cerebral arteries, consistent with occlusive disease. He subsequently continued to experience progressive neurologic deterioration and repeated episodes of cerebral infarction.

He developed squamous cell carcinoma at the age of 41 years that lead to his death at the age of 43 years.

\section{Case 2, Family 2}

A 37-year-old man who had oral thrush, skin lesions with horn formation, nail dystrophy, and repeated pulmonary infections since infancy (Figure 2). At the age

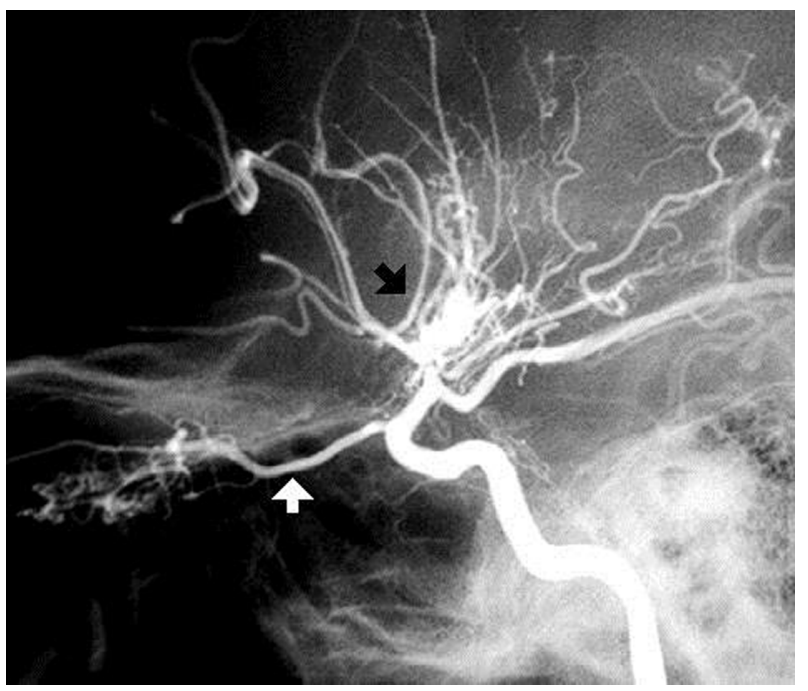

Figure 1: Angiograph performed in case 1 showing arterial dilation (see arrows).

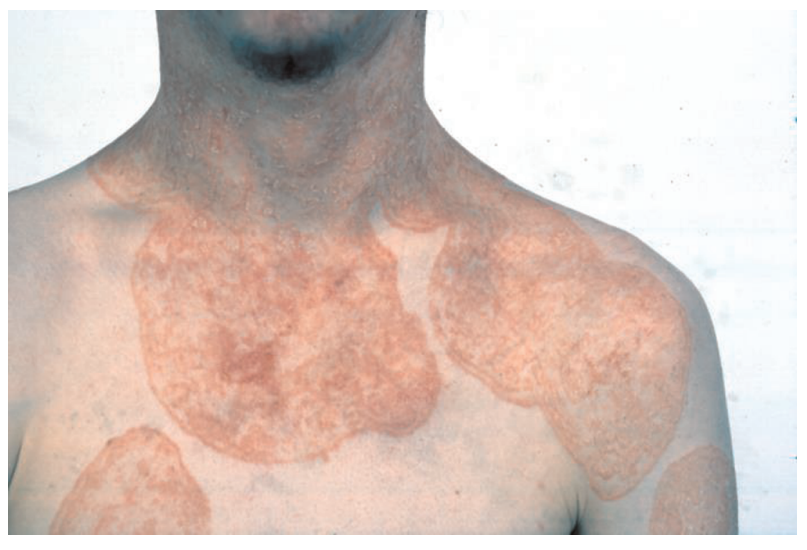

Figure 2: Extensive skin candidiasis with horn formation documented in case 2. 
of 32 years, 25 teeth were extracted because of persistent abscesses.

During his teenage years he developed repeated episodes of cerebral hemorrhage. Angiography showed multiple narrowing and dilation in the basal arteries and the middle cerebral artery branches. He continued to deteriorate neurologically and is currently confined to a wheelchair.

Evaluation of the immune system showed T-cell lymphopenia and normal in vitro response to mitogens, cytomegalovirus, and purified protein derivative (PPD), but no response to Candida antigen. Serum immunoglobulins and specific antibodies were normal.

\section{Case 3, Family 2}

This patient, the son of the case 2 patient, suffered oral thrush since infancy, with mild skin lesions and nail dystrophy. He was diagnosed with hypothyroidism at the age of 12 years. He remains neurologically intact at the age of 14 years.

Evaluation of the immune system revealed a normal number of circulating T cells, B cells, and NK cells. He had normal responses to mitogens but no in-vitro response to the Candida antigen. Immunoglobulins G, $\mathrm{A}$, and $\mathrm{M}$ were normal and responses to immunizations were adequate.

\section{Case 4, Family 3}

A 53-year-old man of Scottish decent who had repeated oral candidiasis that started after birth but intensified over the years. At 14 years of age, he suffered severe esophageal candidiasis with resultant strictures that required dilatation on more than 10 occasions. Strictures led to a significant decrease in oral intake, with resultant weight loss. He had no skin or nail involvement. At the age of 46 years he was diagnosed with hypothyroidism. He had no other infections. Family history revealed hypothyroidism in his mother and sister and oral candidiasis in his daughter (Case 5).

Evaluation of the immune system showed a normal IgG level of $15.6 \mathrm{~g} / \mathrm{L}$, elevated $\operatorname{IgA}$ of 6.0 , and low IgM at $<0.1 \mathrm{~g} / \mathrm{L}$. Lymphocyte markers demonstrated normal numbers of CD19(145), CD3(166), CD4(612), and CD8 (445) positive cells but slightly low NK cells at 46 . Specific antibody levels were protective to rubella, varicella, and tetanus. Proliferative responses to mitogens were normal but in-vitro response to Candida was absent despite extensive exposure.

\section{Case 5, Family 3}

This patient is the 20-year-old daughter of the case 4 patient; she had oral thrush from the age of 3 weeks. These episodes occurred every $2-4$ months and resolved within days after treatment with nystatin or fluconazole.

She had an episode of chickenpox at the age of 1 year; it was uneventful and she tolerated live vaccines with no adverse events. She had fungal nail infections at the age of 6 years and suffered repeated episodes of sinusitis. At the age of 10 years she was diagnosed with hypothyroidism. At the age of 17 years she was diagnosed with strictures in the upper parts of the esophagus. There were also large spots of white mucoid plaques in the distal esophagus, consistent with Candida esophagitis. A culture obtained from esophageal biopsy grew Candida. She frequently suffers from warts.

Evaluation of the immune system revealed elevated serum IgG levels at $19.5 \mathrm{~g} / \mathrm{L}$ but normal IgA of 1.7 and IgM 0.8. Specific antibodies to tetanus, mumps, and rubella were protective, and the response to vaccinations with pneumovax was normal. Lymphocyte immunophenotyping showed 743 CD3+ cells, 876 CD4+ cells, and $673 \mathrm{CD} 8+$ cells. Proliferation in response to mitogens and antigens was normal.

\section{Cases 6-9, Family 4}

The case 6 patient, a 7-year-old male of Irish descent, suffered repeated oral thrush from the age of 4 years. He had no other infections, nor skin or nail lesions. His 37year-old father (case 7) appeared to have suffered from identical symptoms since childhood. Two other children in the family, 4-year-old twins (cases 8 and 9), also presented with oral thrush. All of these patients were found to have a heterozygous mutation in STAT1, whereas the mother and another sibling were normal and had no related symptoms.

Evaluation of immunity showed normal lymphocyte phenotyping as well as in vitro responses to mitogens and antigens. Immunoglobulins and specific antibodies were normal.

\section{Genetic analysis}

All of the patients were found to have heterozygous mutations in STAT1 within the coiled domain. There 
were 4 new mutations in these patients (Figures 3 and 4).

Case 1 had a $\mathrm{C}$ to $\mathrm{T}$ transition at position 863 , which translates into a $\mathrm{T}$ to $\mathrm{I}$ substitution at amino acid

(a) STAT1

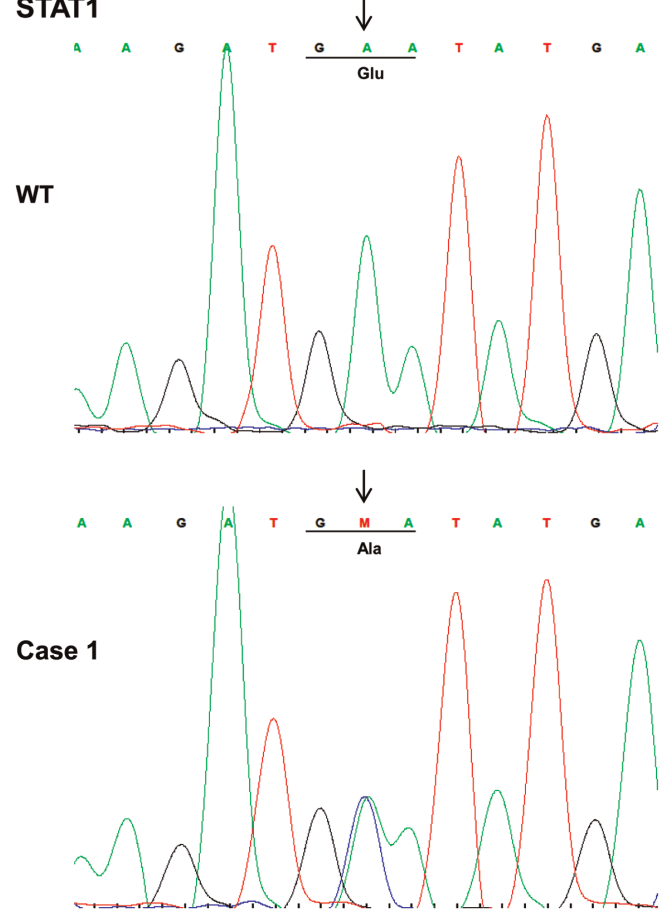

(c)

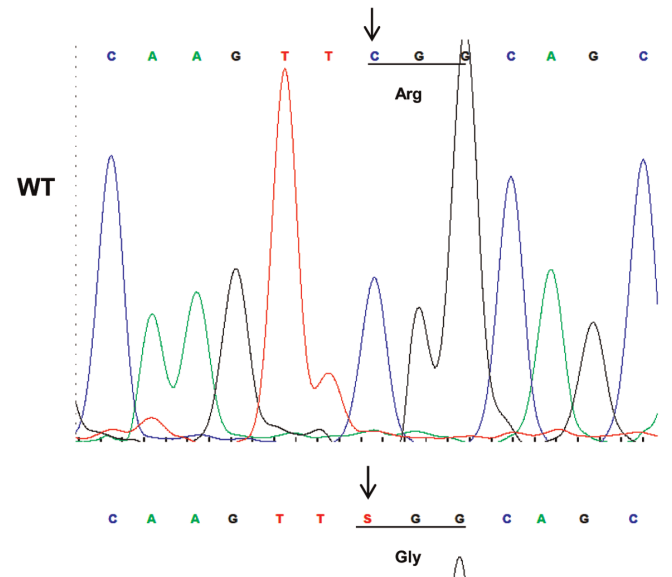

Cases 4, 5

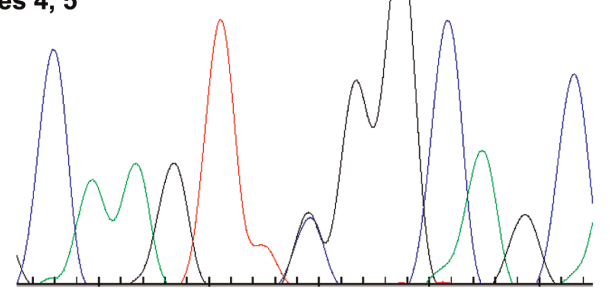

position 288. Another mutation (T288A) in this location has been previously reported (Liu et al. 2011).

Cases 2 and 3 had a $C$ to $\mathrm{G}$ transition at position 820 , which predicts an $\mathrm{R}$ to $\mathrm{G}$ substitution at position 274 .

(b)

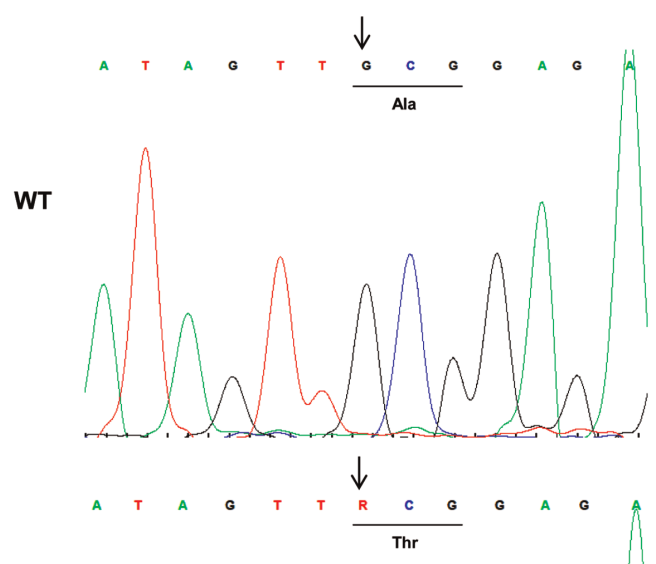

Cases 2, 3

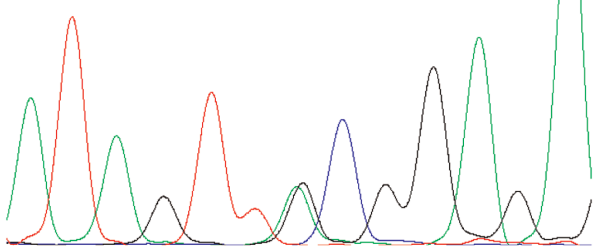

(d)
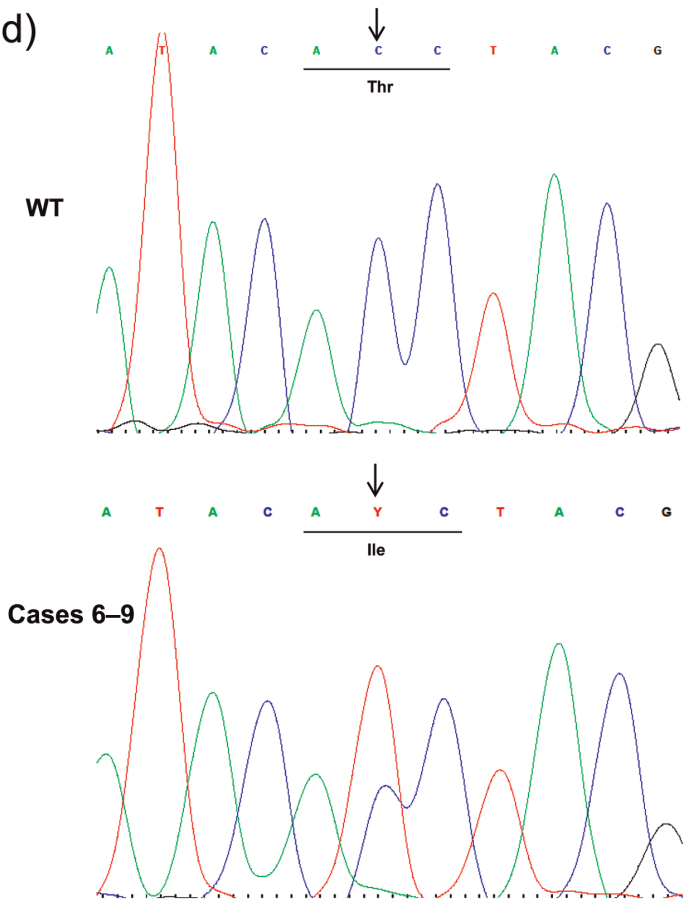

Figure 3: Demonstration of sequence tracings of STAT1 mutations and wild type in: case 1 c. 506A > C (A), cases 2 and 3 c.799G > A (B), cases 4 and 5 c. $820 C>G(C)$, and cases $6-9$ c. $863 C>T(D)$. 


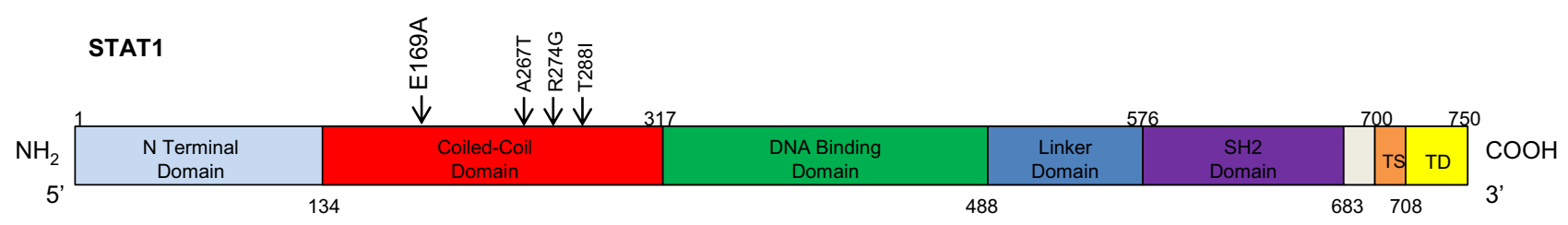

Figure 4: Human STAT1 protein showing the boundaries of the $\mathrm{N}$ terminal domain, coiled-coil domain, DNA binding domain, linker domain, SH2 domain, tail segment domain, and transactivation domain. The location of the E169A, A267T, R274G, and T288I mutations are depicted.

The R274Q mutation was previously reported in this location (Liu et al. 2011).

Cases 4 and 5 had an A to $\mathrm{C}$ transition at position 506 predicting an $\mathrm{E}$ to $\mathrm{A}$ amino acid substitution.

Cases 6-9 (a father and 3 children) were found to have a $G$ to $A$ transition at the position 799 , which predicts an $\mathrm{A}$ to $\mathrm{T}$ amino acid substitution at 267. A different mutation, A267V, was previously reported at the same location (Liu et al. 2011; van de Veerdonk et al. 2011).

\section{Brain vascular anomalies in STAT1}

Analysis of the sections from a brain biopsy of the case 2 patient showed several abnormalities in cerebral cortex and white matter. The pia-arachnoid showed focal dense collagenous thickening often centered around medium-sized arteries whose walls were thickened and largely acellular. Other vessels of the same size contained abundant plump fibroblasts and lymphocytes within the thickened intima and media. Interruption of the elastic lamina could occasionally be identified. Many small arteries within the cortex had thickened walls and a narrow cuff of lymphoctyes. Obliterated blood vessel lumens were rarely seen. Alongside a single pia artery were rare giant cells with several nuclei each. The cerebral cortex showed a scattered increase in the number of microglial cells and had a single microglial nodule with vague degenerative material in the center. No neurofibrillary tangles, ischemic neurons, or neuronal inclusions were seen.

In the white matter there was diffuse chronic reactive astrogliosis and several foci of demyelination with plump, reactive astroglia. The axons were relatively preserved in these foci.

Stains for fungi, acid-fast bacilli, and treponemas were unremarkable.
Electron microscopy was negative for viral inclusion bodies.

The MRI imaging demonstrated multiple infarctions involving the right basal ganglia extending to the subinsular cortex, left basal ganglia, left watershed region, and left cerebellum.

There were 2 focal areas of increased signal in the posteromedial region of the right cerebellar hemisphere measuring 1-2 cm each. There was considerable tortuosity to the basilar artery. There was a crescentic area of increased signal seen within the right lateral edge of the basilar artery, likely representing a thrombus. This study confirmed multiple infarcts and atrophy.

\section{Discussion}

The characterizing features of the patients presented here with heterozygous STAT1 mutations are severe CMCC from early childhood and cerebrovascular abnormalities in the second decade of life that led to occlusive disease, hypothyroidism, malignant transformation of the skin, and in 2 patients there were recurrent sinupulmonary infections without significant detectable reduction in $\mathrm{B}$ - or $\mathrm{T}$-cell functions.

The mutation of STAT1 in these patients resided in the coiled-coil domain, a region previously reported in the literature to harbor several mutations, all with gain of function as demonstrated in an Epstein-Barr virus cell line (Liu et al. 2011). The coiled-coil domain plays a role in STAT dimerization and later on in nuclear dephosphorylation (Chen et al. 1998; Levy and Darnell 2002). These mutations in the coiled-coil domain of STAT1 result in reduced production of IL17, IL22, and INF. Reduced production of IL17, IL22, and INF in the coiled-coil domain of STAT1 results in several gain of function mutations (Marodi et al. 2012).

In a recent study (Sharfe et al. 2014), we described a cohort of patients showing severe progressive combined 
immunodeficiency with some patients succumbing to disease in early adulthood or late childhood; all patients had de novo mutations in the DNA binding domain or near this area of STAT1. Our study of STAT1 activity revealed a clear loss of function (Sharfe et al. 2014). The current group of patients carry mutations in the coiled-coil domain with features resembling a previously described group of patients (Liu et al. 2011; van de Veerdonk et al. 2011) showing at least 2 generations with similar symptoms and genetic defects in an autosomal dominant inheritance fashion. This suggests either a slightly less severe effect on STAT1 activity or a different pathogenic mechanism in these 2 groups that share the hallmark of chronic candidiasis. Although they do not show significant immune compromise and fatal viral infections, the mutations in the coiled-coil domain still carry potential risk for serious morbidity. Some of the patients suffered from vascular events of the central nervous system that left them handicapped. The imaging of the central nervous system of 2 cases showed images typical of Moya-Moya disease. Vascular events occurring in early age in CMCC patients was reported first by Grouhi et al. (1998) and more recently by Liu et al. (2011). From our pathologic investigation of brain lesion sections, it appears that these are foci of ischemia that are secondary to primary vasculitis. Autoimmunity, although not varied in these patients, could be a result of a disruption of regulatory mechanisms or constant inflammation and exposure to selfantigens. The exact mechanism underlying these vascular events is yet to be elucidated.

Two of the patients showed recurrent infections, this could be due to a contribution from other genetic defects or a background attenuating disease severity. Although no major immunologic defects were found in the immune system workup, it may be that some minor functional defects contribute to this propensity.

Two patients in our group and several in Liu et al. (2011) had thyroid disease. In Park et al. (2000), it appeared that thyrotropin induced the SOCS1 protein (suppressor of cytokine signaling 1) and that this molecule affects STAT1 phosphorylation. As suggested by Park et al. (2000), it is possible that defects in STAT1 will alter thyrotropin action in the thyroid gland and lead to destruction or dysfunction of the gland.

One of our patients died of squamous cell carcinoma; from previous reports it appears that squamous cell carcinoma is prevalent in CMCC patients with STAT1 mutations (Liu et al. 2011; van de Veerdonk et al. 2011). The very same neoplastic disease was described in patients with chronic candidiasis such as those with autoimmune polyendocrinopathy-candidiasis-ectodermal dystrophy and autoimmune regulator deficiency (Perheentupa 2006; Rautemaa et al. 2007). Therefore, it is less likely that this is a direct result of STAT1 dysfunction but, rather, a compromise of immune mechanisms, such as Th17 antifungal activity, and a lack of suppression of Candida growth. Candida albicans is known to possess catalytic activity resulting in the production of nitrosamines, products that are carcinogenic and considered to be a significant risk factor for squamous cell carcinoma (Hsia 1981; Krogh 1990); therefore, it is very likely that malignant transformation occurs as a result of uncontrolled Candida proliferation and its products.

We present new mutations in the coiled-coil domain of STAT1 that are clinically different from our previously reported group of patients with mutations in the DNA binding domain (Sharfe et al. 2014). These patients appeared to have 2 severe manifestations, central nervous system vasculitis and squamous cell carcinoma; it is possible that at least the latter could be avoided by rigorous treatment with antifungals. Follow-up of these patients' skin and mucosa conditions and vigorous treatment are crucial and life saving.

\section{REFERENCES}

Boisson-Dupuis, S., Kong, X.F., Okada, S., Cypowyj, S., Puel, A., Abel, L., and Casanova, J.L. 2012. Inborn errors of human STAT1: allelic heterogeneity governs the diversity of immunological and infectious phenotypes. Curr. Opin. Immunol. 24(4):364-378. PMID: 22651901. doi: 10.1016/j.coi.2012.04.011.

Chen, X., Vinkemeier, U., Zhao, Y., Jeruzalmi, D., Darnell, J.E. Jr, and Kuriyan, J. 1998. Crystal structure of a tyrosine phosphorylated STAT-1 dimer bound to DNA. Cell. 93(5):827-839.

Dupuis, S., Jouanguy, E., Al-Hajjar, S., Fieschi, C., AlMohsen, I.Z., Al-Jumaah, S., Yang, K., Chapgier, A., Eidenschenk, C., Eid, P., Al Ghonaium, A., Tufenkeji, H., Frayha, H., Al-Gazlan, S., Al-Rayes, H., Schreiber, R.D., Gresser, I., and Casanova, J.L. 2003. Impaired response to interferon-alpha/beta and lethal viral disease in human STAT1 deficiency. Nat. Genet. 33(3): 388-391.

Grouhi, M., Dalal, I., Nisbet-Brown, E., and Roifman, C.M. 1998. Cerebral vasculitis associated with chronic 
mucocutaneous candidiasis. J. Pediatr. 133(4):571-574. doi: 10.1016/S0022-3476(98)70072-1.

Hsia, C.C., Sun, T.T., Wang, Y.Y., Anderson, L.M., Armstrong, D., and Good, R.A. 1981. Enhancement of formation of the esophageal carcinogen benzylmethylnitrosamine from its precursors by Candida albicans. Proc. Natl. Acad. Sci. USA. 78(3):18781881. doi: 10.1073/pnas.78.3.1878.

Krogh, P. 1990. The role of yeasts in oral cancer by means of endogenous nitrosation. Acta. Odontol. Scand. 48(1): 85-88. doi: 10.3109/00016359009012738.

Levy, D.E., and Darnell, J.E. Jr. 2002. Stats: transcriptional control and biological impact. Nat. Rev. Mol. Cell. Biol. 3(9):651-662. doi: 10.1038/nrm909.

Liu, L., Okada, S., Kong, X.F., Kreins, A.Y., Cypowyj, S., Abhyankar, A., Toubiana, J., Itan, Y., Audry, M., Nitschke, P., Masson, C., Toth, B., Flatot, J., Migaud, M., Chrabieh, M., Kochetkov, Y., Zhao, T., Bolze, A., Borghesi, A., Toulon, A., Hiller, J., Eyerich, S., Eyerich, K., Gulácsy, V., Chernyshova, L., Chernyshov, V., Bondarenko, A., Grimaldo, R.M., Blancas-Galicia, L., Beas, I.M., Roesler, J., Magdorf, K., Engelhard, D., Thumerelle, C., Burgel, P.R., Hoernes, M., Drexel, B., Seger, R., Kusuma, T., Jansson, A.F., SawalleBelohradsky, J., Belohradsky, B., Jouanguy, E., Bustamante, J., Bué, M., Karin, N., Wildbaum, G., Bodemer, C., Lortholary, O., Fischer, A., Blanche, S., Al-Muhsen, S., Reichenbach, J., Kobayashi, M., Rosales, F.E., Lozano, C.T., Kilic, S.S., Oleastro, M., Etzioni, A., Traidl-Hoffmann, C., Renner, E.D., Abel, L., Picard, C., Maródi, L., Boisson-Dupuis, S., Puel, A., and Casanova, J.L. 2011. Gain-of-function human STAT1 mutations impair IL-17 immunity and underlie chronic mucocutaneous candidiasis. J. Exp. Med. 208(8):1635-1648. PMID: 21727188. doi: 10.1084/ jem.20110958.
Marodi, L., Cypowyj, S., Toth, B., Chernyshova, L., Puel, A., and Casanova, J.L. 2012. Molecular mechanisms of mucocutaneous immunity against Candida and Staphylococcus species. J. Allergy. Clin. Immunol. 130:1019-1027. PMID: 23040277. doi: 10.1016/j.jaci. 2012.09.011.

O'Shea, J.J., Holland, S.M., and Staudt, L.M. 2013. JAKs and STATs in immunity, immunodeficiency and cancer. N. Engl. J. Med. 368(2):161-170. PMID: 23301733. doi: 10.1056/NEJMra1202117.

Park, E.S., Kim, H., Suh, J.M., Park, S.J., Kwon, O.Y., Kim, Y.K., Ro, H.K., Cho, B.Y., Chung, J., and Shong, M. 2000. Thyrotropin induces SOCS-1 (suppressor of cytokine signaling-1) and SOCS-3 in FRTL-5 thyroid cells. Mol. Endocrinol. 14(3):440-448.

Perheentupa, J. 2006. Autoimmune polyendocrinopathycandidiasis-ectodermal dystrophy. J. Clin. Endocrinol. Metab. 91(8):2843-2850. doi: 10.1210/jc.2005-2611.

Rautemaa, R., Hietanen, J., Niissalo, S., Pirinen, S., and Perheentupa, J. 2007. Oral and oesophageal squamous cell carcinoma-a complication or component of autoimmune polyendocrinopathy-candidiasis-ectodermal dystrophy (APECED, APS-I). Oral. Oncol. 43(6): 607-613. doi: 10.1016/j.oraloncology.2006.07.005.

Sharfe, N., Nahum, A., Newell, A., Dadi, H., Ngan, B., Pereira, S.L., Herbrick, J.A., and Roifman, C.M. 2014. Fatal combined immunodeficiency associated with heterozygous mutation in STAT1. J. Allergy. Clin. Immunol. 133(3):807-817. doi: 10.1016/j.jaci.2013. 09.032 .

van de Veerdonk, F.L., Plantinga, T.S., Hoischen, A., Smeekens, S.P., Joosten, L.A., Gilissen, C., et al. 2011. STAT1 mutations in autosomal dominant chronic mucocutaneous candidiasis. N. Engl. J. Med. 365(1):54-61. PMID: 21714643. doi: 10.1056/NEJ Moa1100102. 